\title{
A Bayesian nonparametric approach to ecological risk assessment
}

\author{
Guillaume Kon Kam King, Julyan Arbel and Igor Prünster
}

\begin{abstract}
We revisit a classical method for ecological risk assessment, the Species Sensitivity Distribution (SSD) approach, in a Bayesian nonparametric framework. SSD is a mandatory diagnostic required by environmental regulatory bodies from the European Union, the United States, Australia, China etc. Yet, it is subject to much scientific criticism, notably concerning a historically debated parametric assumption for modelling species variability. Tackling the problem using nonparametric mixture models, it is possible to shed this parametric assumption and build a statistically sounder basis for SSD. We use Normalized Random Measures with Independent Increments (NRMI) as the mixing measure because they offer a greater flexibility than the Dirichlet process. Indeed, NRMI can induce a prior on the number of components in the mixture model that is less informative than the Dirichlet process. This feature is consistent with the fact that SSD practitioners do not usually have a strong prior belief on the number of components. In this short paper, we illustrate the advantage of the nonparametric SSD over the classical normal SSD and a kernel density estimate SSD on several real datasets. We summarise the results of the complete study in Kon Kam King et al. (2016), where the method is generalised to censored data and a systematic comparison on simulated data is also presented, along with a study of the clustering induced by the mixture model to examine patterns in species sensitivity.
\end{abstract}

Keywords: Bayesian Nonparametrics, Ecotoxicology, $\mathrm{HC}_{5}$, Mixture models, Normalized random measures, Species Sensitivity Distribution.

Guillaume Kon Kam King

University of Torino, Italy and Collegio Carlo Alberto, Moncalieri, Italy, e-mail: guillaume.konkamking. work@gmail.com

Julyan Arbel

Inria Grenoble - Rhône-Alpes \& Université Grenoble Alpes, France, e-mail: julyan. arbel@inria.fr

Igor Prünster

Department of Decision Sciences, BIDSA and IGIER, Bocconi University, Italy, e-mail: igor@unibocconi.it 


\section{Introduction}

Assessing the response of a community of species to an environmental stress is critical for ecological risk assessment. Methods for this purpose vary in levels of complexity and realism. Species Sensitivity Distribution (SSD) represents an intermediate tier, more refined than rudimentary assessment factors (Posthuma et al., 2002) but practical enough for routine use by environmental managers and regulators in most developed countries (Australia, Canada, China, EU, South Africa, USA...). The SSD approach is intended to provide, for a given contaminant, a description of the tolerance of all species possibly exposed using information collected on a sample of those species. This information consists of Critical Effect Concentrations (CECs), a concentration specific to a species which marks a limit over which the species suffers a critical level of effect. This is for instance the concentration at which $50 \%$ of the tested organisms died (Lethal Concentration $50 \%\left(\mathrm{LC}_{50}\right)$ ), or the concentration which inhibited growth or reproduction by $50 \%$ compared to the control experiment (Effect Concentration 50\% ( $\left.\mathrm{EC}_{50}\right)$ ). Each CEC is the summary of long and costly bioassay experiments for a single species, so they are rarely available in large number. Typical sample sizes range from 10 to 15 (ECHA, 2008).

To describe the tolerance of all species to be protected, the distribution of the CECs is then estimated from the sample. In practice, a parametric distributional assumption is often adopted (Forbes and Calow, 2002): the CECs are assumed to follow a log-normal (Aldenberg and Jaworska, 2000), log-logistic (Kooijman, 1987), triangular (Van Straalen, 2002; Zhao and Chen, 2016) or BurrIII (Shao, 2000) distribution.

Once the response of the community is characterised by the distribution, the goal of risk assessment is to define a safe concentration protecting all or

most of the species. In the case of distributions without a lower threshold strictly above 0, a cut-off value is often chosen as the safe concentration. Typically, this is the Hazardous Concentration for $5 \%$ of the Species $\left(\mathrm{HC}_{5}\right)$, which is the 5 th percentile of the distribution. Reasonings behind this choice include: that the lowest bound of the confidence interval around the 5th percentile will be used instead of the estimate, that a safety factor will be subsequently applied to that value and that ecosystems have a certain resilience to perturbations.

The lack of justification for the choice of any given parametric distribution has sparked several research directions. Some authors (Xu et al., 2015; He et al., 2014; Jagoe and Newman, 1997; Van Straalen, 2002; Xing et al., 2014; Zhao and Chen, 2016) have sought to find the best parametric distribution by model comparison using goodness-of-fit measures. The general understanding is that no single distribution seems to provide a superior fit and that the answer is dataset dependent (Forbes and Calow, 2002). Therefore, the log-normal distribution has become the customary choice, notably because it readily provides confidence intervals on the $\mathrm{HC}_{5}$, and because model com- 
parison and goodness of fit tests have relatively low power on small datasets, precluding the emergence of a definite answer to the question. Another research direction consisted in seeking to avoid any reference to a distribution, using so-called nonparametric or distribution-free approaches. Those efforts included using the empirical distribution function (Suter II et al., 1999; Jones et al., 1999), methods based on ranks (Van Der Hoeven, 2001; Chen, 2004), bootstrap resampling (Jagoe and Newman, 1997; Wang et al., 2008) or nonparametric kernel density estimation (Wang et al., 2015). All these approaches have in common that they require large sample sizes to be effectively applicable. Finally, authors have considered the possibility that the distribution of the CECs might not be a single distribution but rather a mixture of distributions (Zajdlik et al., 2009), datasets being an assemblage of several log-normally distributed subgroups (Kefford et al., 2012; Craig, 2013). This is more realistic from an ecological point of view because several factors influence the tolerance of a species to a contaminant such as the taxonomic group or the mode of action, and contaminant such as pesticides might even target specific species groups. Therefore, there is strong evidence in favour of the presence of groups of CECs, although the CECs within a group might remain log-normally distributed.

Ignorance of the group structure is a strong motivation for a nonparametric approach. However, the method must remain applicable to small datasets, which suggests trying to improve on the existing frequentist nonparametric methods. Bayesian nonparametric mixture models offer an interesting solution for both large and small datasets, because the complexity of the mixture model adapts to the size of the dataset. It offers a good compromise between a simplistic one-component parametric model and a kernel density method which in a certain sense lacks flexibility and might cause overfitting. Moreover, the low amount of information available in small datasets to estimate the groups parameters can be complemented via the prior, as some a priori degree of information is generally available from other species or contaminants (Awkerman et al., 2008; Craig, 2013; Craig et al., 2012). This paper summarises the results of the complete study in Kon Kam King et al. (2016).

The rest of the article is organised as follows. In Section 2 we present the Bayesian nonparametric (BNP) model and existing frequentist models for SSD and explain how to obtain a density estimate. Then in Section 3 we compare the different methods on a real dataset, illustrating the benefits of the BNP SSD. We conclude with a final discussion in Section 4.

\section{Models for SSD}

Given that concentrations vary on a wide range, it is common practice to work on log-transformed concentrations. Consider a sample of $n$ log-concentrations denoted by $\boldsymbol{X}=\left(X_{1}, \ldots, X_{n}\right)$. We propose to carry out density estimation for the SSD based on sample $\boldsymbol{X}$ by use of nonparametric mixtures. Bayesian 
nonparametric mixtures were introduced in Lo (1984) with Dirichlet process mixtures (DPM). Generalizations of the DPM correspond to allowing the mixing distribution to be any discrete nonparametric prior. A large class of such prior distributions is obtained by normalizing increasing additive processes (Sato, 1999). The normalization step, under suitable conditions, gives rise to so-called normalized measures with independent increments (NRMI) as defined by Regazzini et al. (2003), see also Barrios et al. (2013) for a recent review. An NRMI mixture model is defined hierarchically as:

$$
\begin{aligned}
X_{i} \mid \mu_{i}, \sigma & \stackrel{\text { ind }}{\sim} k\left(\cdot \mid \mu_{i}, \sigma\right), \quad \mu_{i} \mid \tilde{P} \stackrel{\text { i.i.d. }}{\sim} \tilde{P}, \quad i=1, \ldots, n, \\
\tilde{P} & \sim \mathrm{NRMI}, \quad \sigma \sim \operatorname{Ga}\left(a_{\sigma}, b_{\sigma}\right) .
\end{aligned}
$$

where $k$ is a kernel, which we assume parametrized by some $\theta=(\mu, \sigma) \in$ $\mathbb{R} \times \mathbb{R}_{+}$, and $\tilde{P}$ is a random probability on $\mathbb{R}$ whose distribution is an NRMI. In our model, all clusters have a common variance. This is easier to fit on a small dataset, because information about the variance is pooled across clusters. Similar mixture SSD models described in Craig (2013) also assume common variance. As described in the Introduction, concentrations are commonly fitted with a log-normal distribution. Our aim is to move from this parametric model to the nonparametric one in (1). In order to allow comparisons to be made, we stick to the normal specification for $k$ on the log-concentrations $\boldsymbol{X}$ by letting: $k(x \mid \mu, \sigma)=\mathcal{N}(x \mid \mu, \sigma)$. Under this framework, density estimation is carried out by evaluating the posterior predictive density along the lines of Barrios et al. (2013):

$$
\hat{f}(x \mid \tilde{P}, \boldsymbol{X})=\iint k(x \mid \mu, \sigma) \mathrm{d} \pi(\sigma) \mathrm{d} \tilde{P}(\mu)
$$

for any $x$ in $\mathbb{R}$, where $\pi$ denotes the posterior distribution of $\sigma$.

To specify the prior, we choose as mixing random measure the normalized stable process (Kingman, 1975) with:

i a stability parameter $\gamma=0.4$, which controls the flatness of the prior on the number of clusters. The parameter $\gamma$ can take values in $(0,1)$. Taking the limit $\gamma \rightarrow 0$ reduces the model to a Dirichlet process, larger values of $\gamma$ lead to less informative priors on the number of clusters. The parameter $\gamma$ was chosen as a good compromise between model flexibility and numerical stability. The total mass parameter is, without loss of generality, set equal to 1 .

ii a base measure (which corresponds to the mean of the random probability measure) $P_{0}(\cdot)=\mathcal{N}\left(\cdot \mid \varphi_{1}, \varphi_{2}\right)$ with mean $\varphi_{1}$ and standard deviation $\varphi_{2}$, hyperparameters fixed a priori to specify a certain knowledge in the degree of smoothness

iii a common variance for all the clusters with a vaguely informative prior distribution $G a(0.5,0.5)$. 
Recent years have witnessed the appearance of a wealth of softwares dedicated to implement Bayesian nonparametric models and sample from their posterior. To cite a few, the R package DPpackage (Jara et al., 2011), is a rather comprehensive bundle of functions for Bayesian nonparametric models, while Bayesian Regression (Karabatsos, 2016) is a software for Bayesian nonparametric regression. For posterior sampling, we use the R package BNPdensity and the function MixNRMI1 which implements BNP density models under a general specification of normalized random measures based on the generalised gamma processes (see Barrios et al., 2013). The package is available from the Comprehensive R Archive Network (CRAN).

To illustrate the interest of the Bayesian nonparametric SSD, we compare our proposed BNP model to two commonly used frequentist models: the normal distribution (Aldenberg and Jaworska, 2000) and the nonparametric Kernel Density Estimate (KDE) recently proposed by Wang et al. (2015). For both frequentist approaches, the data is assumed to be iid. Density estimates take on respectively the following form ( $\hat{\mu}$ and $\hat{\sigma}$ are MLE)

$$
\hat{f}_{\mathcal{N}}(x)=\mathcal{N}(x \mid \hat{\mu}, \hat{\sigma}) \quad \text { and } \quad \hat{f}_{K D E}(x)=\frac{1}{n} \sum_{i=1}^{n} \mathcal{N}\left(x \mid X_{i}, 1.06 \hat{\sigma} n^{-\frac{1}{5}}\right) .
$$

\subsection{Model comparison and cross-validation}

For the purpose of comparing the predictive performance of the model, we resort to Leave-One-Out (LOO) cross-validation. We compute the LOOs for each of the methods as $\forall i, \mathrm{LOO}_{i}=\hat{f}\left(X_{i} \mid \boldsymbol{X}_{-i}\right)$ where $\hat{f}\left(x \mid \boldsymbol{X}_{-i}\right)$ is the density for one of the three methods estimated from $\boldsymbol{X}$ with $X_{i}$ left out. The LOOs for the BNP model correspond to the conditional predictive ordinates (CPOs) statistics which are commonly used in applications, see Gelfand (1996). A $\mathrm{CPO}$ statistic is defined for each log-concentration $X_{i}$ as follows:

$$
\mathrm{CPO}_{i}=\hat{f}\left(X_{i} \mid \boldsymbol{X}_{-i}\right)=\int k\left(X_{i} \mid \theta\right) \mathrm{d} \pi\left(\theta \mid \boldsymbol{X}_{-i}\right)
$$

where $\boldsymbol{X}_{-i}$ denotes the all sample $\boldsymbol{X}$ but $X_{i}, \mathrm{~d} \pi\left(\theta \mid \boldsymbol{X}_{-i}\right)$ is the posterior distribution associated to $\boldsymbol{X}_{-i}$ and $\hat{f}$ is the (cross-validated) posterior predictive distribution of Equation (2). As shown by Barrios et al. (2013), CPOs can be easily approximated by Monte Carlo as

$$
\widehat{\mathrm{CPO}_{i}}=\left(\frac{1}{T} \sum_{t=1}^{T} \frac{1}{k\left(X_{i} \mid \theta^{(t)}\right)}\right)^{-1}
$$

where $\left\{\theta^{(t)}, t=1,2, \ldots, T\right\}$ is an MCMC sample from the posterior distribution. 


\subsection{Quantile estimation and $\mathrm{HC}_{5}$}

The quantity of interest for ecological risk assessment is the $\mathrm{HC}_{5}$, which corresponds to the 5th percentile of the SSD distribution. We choose as an estimator the median of the posterior distribution of the 5th percentile, while the $95 \%$ credible bands are formed by the $2.5 \%$ and $97.5 \%$ quantiles of the posterior distribution of the 5 th percentile. The 5 th percentile of the KDE is obtained by numerical inversion of the cumulative distribution function, and the confidence intervals using nonparametric bootstrap. The 5 th percentile of the normal SSD and its confidence intervals are obtained following the classical method of Aldenberg and Jaworska (2000).

\section{Application to real data}

We applied this model to a selection of contaminants extracted from a large database collected by the National Institute for Public Health and the Environment (RIVM). This database was prepared, studied and published by Hickey et al. (2012). We only considered non censored data, left or right censored data were discarded, while interval censored data were replaced by the centre of the interval. Kon Kam King et al. (2016) will describe how the method can be adapted to include censored data. Using a continuous distribution for the CECs implies that the model does not support ties (or, in other words, observing ties has zero probability). However, ties may appear in the dataset due to the rounding of concentrations. Hence, we used a small jittering of the data.

We selected three example datasets which feature three typical sample sizes: a relatively large carbaryl dataset (CAS: 63-25-2, insecticide, 55 species), a medium-sized temephos dataset (CAS: 3383-96-8, mosquito larvicide, 21 species), and a small captan dataset (CAS: 133-06-2, fungicide, 13 species). Datasets for new contaminants are always small, the minimum requirement set by the European Chemical Agency being 10 species. The datasets can be visualised on the histograms of Figure 1 (left panel).

These datasets illustrate different features of the three approaches: when there is a clear multimodality in the data, the BNP SSD is more flexible than the fixed bandwidth KDE SSD (Figure 1, carbaryl and captan). When the data do not exhibit strong multimodality, as for temephos, the BNP reduces to the normal SSD model, whereas the KDE remains by construction a mixture of many normal components.

One might think to increase the flexibility of the KDE by simply decreasing the bandwidth. However, that would also decrease the robustness of the method. On the middle panel of Figure 1, the LOOs give an indication of the robustness to over-fitting of the three methods. For carbaryl and captan, they show that the superior flexibility of the BNP SSD compared to the KDE $\mathrm{SSD}$ does not come at the expense of robustness, because the median CPO 

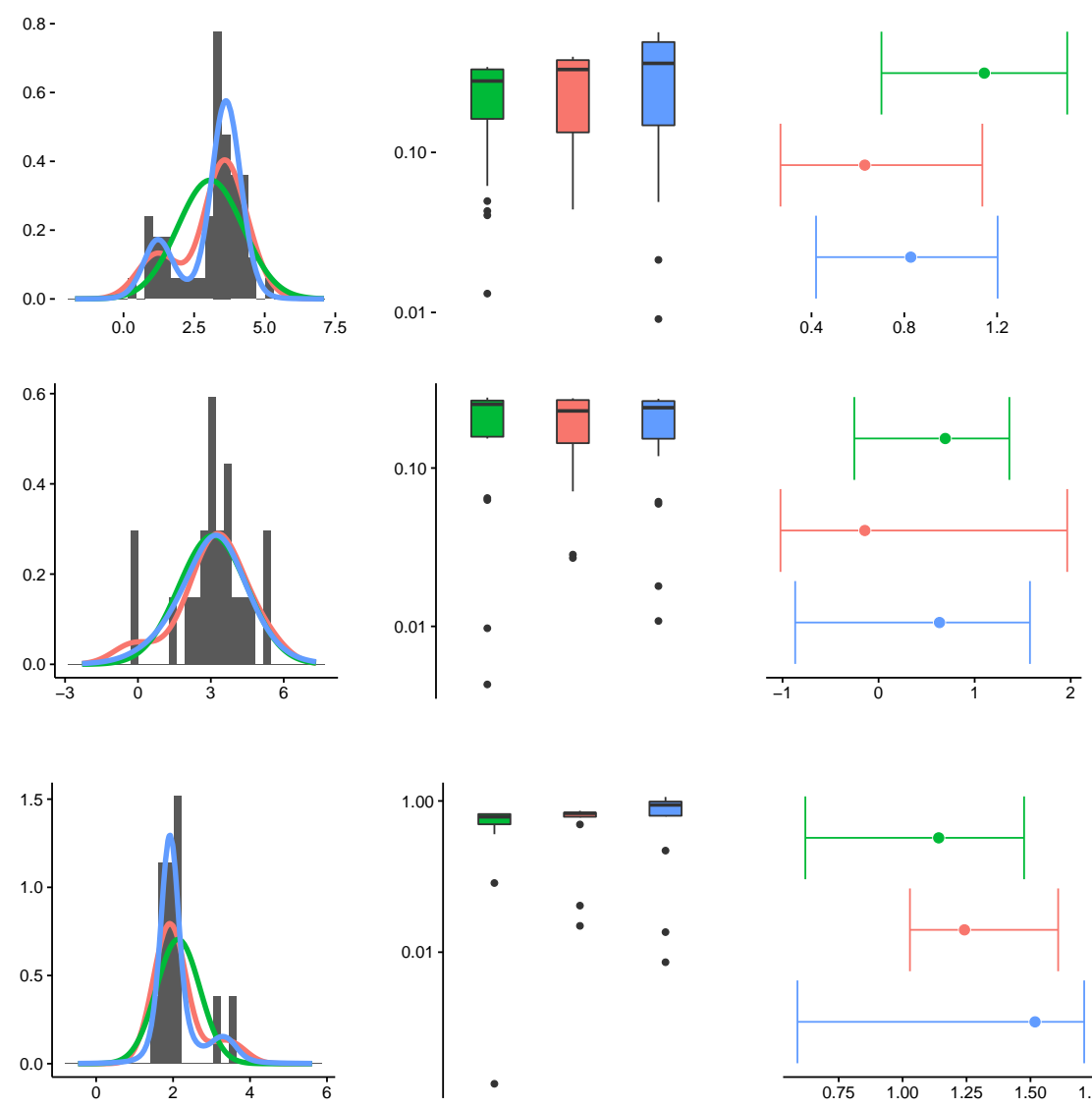

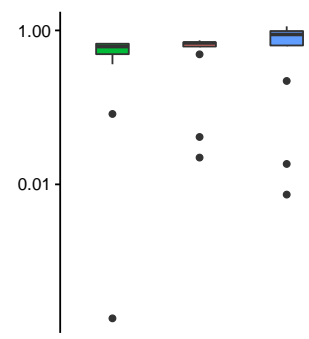

(b) LOO and CPO

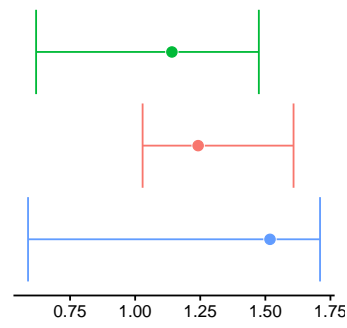

(c) $\log \mathrm{HC}_{5}$

Fig. 1: The top panel represents large-size carbaryl dataset, the middle panel represents the medium-sized temephos dataset, the bottom panel represents small-sized captan dataset. Fits of the Normal (in green), KDE (in red) and BNP (in blue) models. Concentrations are log transformed.

Left: Histogram and density estimates.

Centre: Boxplot for the LOOs (for Normal and KDE) and the CPO (for BNP) on logarithmic scale. The horizontal line corresponds to the median. The box hinges extend to the inner quartiles. The whiskers extend to cover points up to one and a half times the inter-quartile distance away from the hinges. For both frequentist methods, the $\mathrm{n}$ LOOs are obtained by fitting the model $n$ times, while an analytical expression is available for the BNP method (Equation 5).

Right: $\log \mathrm{HC}_{5}$ and associated confidence/credible intervals (for Normal, $\mathrm{KDE}$ and BNP). 
of the BNP SSD is higher than the other two. In the case of temephos, the median LOO likelihood estimate of the normal model is very similar to the median CPO for the BNP SSD, sign that there is little over-fitting. This generally illustrates the fact that model complexity in a BNP model scales with the amount and structure of the data. On the right panel of Figure 1, the credible intervals of the $\mathrm{HC}_{5}$ for the BNP SSD are generally larger than the confidence interval of the normal SSD, which is coherent with the model uncertainty of the nonparametric approach.

\section{Discussion}

The BNP SSD seems to perform well when the dataset deviates from a normal distribution. Its great flexibility is an asset to describe the variability of the data, while it does not seem prone to over-fitting. It can be thought of as an intermediate model between the normal SSD with a single component on the one hand, and the KDE which counts as many components as there are species on the other hand. We chose to base the BNP SSD on NRMI rather than on the more common Dirichlet Process, because it is more robust in case of misspecification of the number of clusters (Lijoi et al., 2007; Barrios et al., 2013). The BNP SSD provides several benefits for risk assessment: it is an effective and robust standard model which adapts to many datasets. Moreover, it readily provides credible intervals. While it is always possible to obtain confidence intervals for a frequentist method using bootstrap, it can be difficult to stabilise the interval for small datasets even with a large number of bootstrap samples. As such, the BNP SSD represents a safe tool to remove one of the arbitrary parametric assumptions of SSD (Forbes and Calow, 2002).

The extended paper supporting the BNP SSD (Kon Kam King et al., 2016) will include a comparison of methods on simulated data, an extension to the case of censored data and an emphasis on the potential benefits of the approach from a biological point of view.

Acknowledgements The authors would like to thank two anonymous reviewers for suggesting several clarifications to the article. J. Arbel was a postdoc at Bocconi University and Collegio Carlo Alberto, Italy, when this article was submitted. J. Arbel and I. Prünster are supported by the European Research Council (ERC) through StG "N-BNP" 306406. 


\section{References}

Aldenberg, T. and Jaworska, J. S. (2000). Uncertainty of the hazardous concentration and fraction affected for normal species sensitivity distributions. Ecotoxicology and environmental safety, 46(1):1-18.

Awkerman, J. A., Raimondo, S., and Barron, M. G. (2008). Development of species sensitivity distributions for wildlife using interspecies toxicity correlation models. Environmental Science and Technology, 42(9):34473452 .

Barrios, E., Lijoi, A., Nieto-Barajas, L. E., and Prünster, I. (2013). Modeling with Normalized Random Measure Mixture Models. Statistical Science, 28(3):313-334.

Chen, L. (2004). A conservative, nonparametric estimator for the 5th percentile of the species sensitivity distributions. Journal of Statistical Planning and Inference, 123(2):243-258.

Craig, P. S. (2013). Exploring novel ways of using species sensitivity distributions to establish PNECs for industrial chemicals: Final report to Project Steering Group. Technical report.

Craig, P. S., Hickey, G. L., Luttik, R., and Hart, A. (2012). Species nonexchangeability in probabilistic ecotoxicological risk assessment. Journal of the Royal Statistical Society. Series A: Statistics in Society, 175(1):243262.

ECHA (2008). Characterisation of dose concentration-response for environment. In Guidance on information requirements and chemical safety assessment, number May, chapter R.10. European Chemicals Agency, Helsinki.

Forbes, V. E. and Calow, P. (2002). Species Sensitivity Distributions Revisited: A Critical Appraisal. Human and Ecological Risk Assessment, $8(3): 473-492$.

Gelfand, A. E. (1996). Model determination using sampling-based methods. Markov chain Monte Carlo in practice, pages 145-161.

He, W., Qin, N., Kong, X., Liu, W., Wu, W., He, Q., Yang, C., Jiang, Y., Wang, Q., Yang, B., and Xu, F. (2014). Ecological risk assessment and priority setting for typical toxic pollutants in the water from Beijing-TianjinBohai area using Bayesian matbugs calculator (BMC). Ecological Indicators, 45:209-218.

Hickey, G. L., Craig, P. S., Luttik, R., and de Zwart, D. (2012). On the quantification of intertest variability in ecotoxicity data with application to species sensitivity distributions. Environmental Toxicology and Chemistry, 31(8):1903-1910.

Jagoe, R. H. and Newman, M. C. (1997). Bootstrap estimation of community NOEC values. Ecotoxicology, 6(5):293-306.

Jara, A., Hanson, T. E., Quintana, F. A., Müller, P., and Rosner, G. L. (2011). DPpackage: Bayesian Semi- and Nonparametric Modeling in R. Journal of Statistical Software, 40(5):1. 
Jones, D. S., Barnthouse, L. W., Suter II, G. W., Efroymson, R. A., Field, J. M., and Beauchamp, J. J. (1999). Ecological risk assessment in a large river-reservoir: 3. Benthic invertebrates. Environmental Toxicology and Chemistry, 18(4):599-609.

Karabatsos, G. (2016). A menu-driven software package of Bayesian nonparametric (and parametric) mixed models for regression analysis and density estimation. Behavior research methods, pages 1-28.

Kefford, B. J., Hickey, G. L., Gasith, A., Ben-David, E., Dunlop, J. E., Palmer, C. G., Allan, K., Choy, S. C., and Piscart, C. (2012). Global scale variation in the salinity sensitivity of riverine macroinvertebrates: Eastern Australia, France, Israel and South Africa. PLoS ONE, 7(5):e35224.

Kingman, J. (1975). Random discrete distributions. Journal of the Royal Statistical Society. Series B, 37(1):1-22.

Kon Kam King, G., Arbel, J., and Prünster, I. (2016). Species Sensitivity Distribution revisited: a Bayesian nonparametric approach. In preparation.

Kooijman, S. (1987). A safety factor for LC 50 values allowing for differences in sensitivity among species. Water Research, 21(3):269-276.

Lijoi, A., Mena, R. H., and Prünster, I. (2007). Controlling the reinforcement in Bayesian non-parametric mixture models. Journal of the Royal Statistical Society. Series B: Statistical Methodology, 69(4):715-740.

Lo, A. Y. (1984). On a Class of Bayesian Nonparametric Estimates: I. Density Estimates. The Annals of Statistics, 12(1):351-357.

Posthuma, L., Suter II, G. W., and Trass, P. T. (2002). Species sensitivity distributions in ecotoxicology. CRC press.

Regazzini, E., Lijoi, A., and Prünster, I. (2003). Distributional results for means of normalized random measures with independent increments. Annals of Statistics, 31(2):560-585.

Sato, K. (1999). Lévy Processes and Infinitely Divisible Distributions (Cambridge Studies in Advanced Mathematics 68, Cambridge University Press, Cambridge).

Shao, Q. (2000). Estimation for hazardous concentrations based on NOEC toxicity data: An alternative approach. Environmetrics, 11(5):583-595.

Suter II, G. W., Barnthouse, L. W., Efroymson, R. A., and Jager, H. (1999). Ecological Risk Assessment in a Large River-Reservoir: 2. Fish Community. Environmental Toxicology and Chemistry, 18(4):589-598.

Van Der Hoeven, N. (2001). Estimating the 5-percentile of the species sensitivity distributions without any assumptions about the distribution. Ecotoxicology, 10(1):25-34.

Van Straalen, N. M. (2002). Threshold models for species sensitivity distributions applied to aquatic risk assessment for zinc. Environmental Toxicology and Pharmacology, 11(3-4):167-172.

Wang, B., Yu, G., Huang, J., and Hu, H. (2008). Development of species sensitivity distributions and estimation of $\mathrm{HC}(5)$ of organochlorine pesticides with five statistical approaches. Ecotoxicology, 17(8):716-724. 
Wang, Y., Wu, F., Giesy, J. P., Feng, C., Liu, Y., Qin, N., and Zhao, Y. (2015). Non-parametric kernel density estimation of species sensitivity distributions in developing water quality criteria of metals. Environmental Science and Pollution Research, 22(18):13980-13989.

Xing, L., Liu, H., Zhang, X., Hecker, M., Giesy, J. P., and Yu, H. (2014). A comparison of statistical methods for deriving freshwater quality criteria for the protection of aquatic organisms. Environmental Science and Pollution Research, 21(1):159-167.

Xu, F.-L., Li, Y.-L., Wang, Y., He, W., Kong, X.-Z., Qin, N., Liu, W.-X., Wu, W.-J., and Jorgensen, S. E. (2015). Key issues for the development and application of the species sensitivity distribution (SSD) model for ecological risk assessment. Ecological Indicators, 54:227-237.

Zajdlik, B. A., Dixon, D. G., and Stephenson, G. (2009). Estimating Water Quality Guidelines for Environmental Contaminants Using Multimodal Species Sensitivity Distributions: A Case Study with Atrazine. Human and Ecological Risk Assessment, 15(3):554-564.

Zhao, J. and Chen, B. (2016). Species sensitivity distribution for chlorpyrifos to aquatic organisms: Model choice and sample size. Ecotoxicology and Environmental Safety, 125:161-9. 